\title{
Gamma Knife surgery for tumor-related trigeminal neuralgia: targeting both the tumor and the trigeminal root exit zone in a single session
}

\author{
Sung Kwon Kim, MD, Dong Gyu Kim, MD, PhD, Young-Bem Se, MD, Jin Wook Kim, MD, PhD, \\ Yong Hwy Kim, MD, Hyun-Tai Chung, PhD, and Sun Ha Paek, MD, PhD
}

Department of Neurosurgery, Seoul National University College of Medicine, Seoul National University Hospital, Seoul, Republic of Korea

\begin{abstract}
OBJECTIVE Gamma Knife surgery (GKS) represents an alternative treatment for patients with tumor-related trigeminal neuralgia (TRTN). However, in previous studies, the primary GKS target was limited to mass lesions. The authors evaluated whether GKS could target both the tumor and the trigeminal root exit zone (REZ) in a single session while providing durable pain relief and minimizing radiation dose-related complications for TRTN patients.
\end{abstract}

METHODS The authors' institutional review board approved the retrospective analysis of data from 15 consecutive patients (6 men and 9 women, median age 67 years, range 45-79 years) with TRTN who had undergone GKS. In all cases, the radiation was delivered in a single session targeting both the tumor and trigeminal REZ. The authors assessed the clinical outcomes, including the extent of pain relief, durability of the treatment response, and complications. Radiation doses to organs at risk (OARs), including the brainstem and the cranial nerve VII-VIII complex, were analyzed as doses received by $2 \%$ or $50 \%$ of the tissue volume and the tissue volume covered by a dose of $12 \mathrm{~Gy}\left(\mathrm{~V}_{12 \mathrm{y}_{\mathrm{y}}}\right)$.

RESULTS The median length of clinical follow-up was 38 months (range 12-78 months). Pain relief with GKS was initially achieved in 14 patients (93.3\%) and at the last follow-up in 13 patients (86.7\%). The actuarial recurrence-free survival rates were $93 \%, 83 \%$, and $69 \%$ at 1,3 , and 5 years after GKS, respectively. Persistent facial numbness was observed in 3 patients $(20.0 \%)$. There were no complications such as facial weakness, altered taste function, hearing impairment, and balance difficulties indicating impaired function of the cranial nerve VII-VIII complex. The $\mathrm{V}_{126 \mathrm{y}}$ in the brainstem was less than or equal to $0.24 \mathrm{~cm}^{3}$ in all patients. There were no significant differences in any OAR values in the brainstem between patients with and without facial numbness after GKS.

CONCLUSIONS The strategy of performing GKS for both tumor and trigeminal REZ in a single session is a safe and effective radiosurgical approach that achieves durable pain control for TRTN patients.

http://thejns.org/doi/abs/10.3171/2015.7.JNS15451

KEY WORDS trigeminal neuralgia; secondary trigeminal neuralgia; tumor-related trigeminal neuralgia; organs at risk; Gamma Knife; pain; stereotactic radiosurgery; $V_{12 G y}$

$\mathrm{T}$ UMOR-Related trigeminal neuralgia (TRTN) is neuralgic pain caused by a tumor adjacent to the components of the fifth cranial nerve (CN); TRTN is present in approximately $1 \%-13 \%$ of patients with trigeminal neuralgia (TN).-2,13,15 Unlike for the management of idiopathic TN, the goal of treating patients with TRTN is to control the tumor and relieve neuralgic pain. Resection of an adjacent tumor on the trigeminal nerve should be considered the first option and may provide excellent neuralgic pain relief if the patients' conditions are clini- cally favorable for resection. ${ }^{2,4,12}$ Stereotactic radiosurgery (SRS) is an alternative approach for managing TRTN and avoids invasive surgery in select patients such as those who have reservations about resection, medical comorbidities, or inoperable tumor characteristics.

Many studies have reported that SRS controls pain well and results in good long-term outcomes in TRTN patients. ${ }^{8,10,14,16,21,23}$ The primary target in all previous studies examining SRS for TRTN was the adjacent mass lesion causing the TN. When no pain relief or recurrent pain after

ABBREVIATIONS BNI = Barrow Neurological Institute; $\mathrm{CN}=$ cranial nerve; $\mathrm{D}_{\mathrm{x} \%}=$ dose received by $\mathrm{x} \%$ of the tissue volume; GKS = Gamma Knife surgery; OAR = organ at risk; REZ = root exit zone; $\mathrm{SRS}$ = stereotactic radiosurgery; TN = trigeminal neuralgia; TRTN = tumor-related trigeminal neuralgia; $\mathrm{V}_{12 \mathrm{~Gy}}=$ volume covered by 12 Gy. SUBMITTED February 26, 2015. ACCEPTED July 20, 2015.

INCLUDE WHEN CITING Published online January 22, 2016; DOI: 10.3171/2015.7.JNS15451. 
an SRS targeting the mass lesion is observed during the follow-up period, repeat SRS targeting the trigeminal root exit zone (REZ) or trigeminal nerve may be considered to achieve additional pain relief; for example, SRS may also be used to target idiopathic TN. ${ }^{8}$

However, repeat SRS can be a cumbersome procedure that may place a substantial economic burden on the patient and society. ${ }^{19}$ Furthermore, pain recurrence has been reported in approximately $24 \%-47 \%$ of TRTN patients after SRS targeting of only the mass lesion. .,10,14,21,23 $^{2}$ Therefore, we examined the outcomes of an SRS method in which both the tumor and trigeminal REZ were targeted in a single-session SRS for TRTN. To our knowledge, no previous reports have described this approach. This lack of data reflects the belief of clinicians, without definite evidence, that radiation-related complications may be more severe because of the effect of the larger radiation dose on adjacent vital structures, including the brainstem, facial nerves, and vestibulocochlear nerves, when both the tumor and trigeminal REZ are targeted in a single-session SRS.

This study retrospectively analyzed the outcomes in patients with TRTN who underwent Gamma Knife surgery (GKS) targeting both the tumor and trigeminal REZ in a single session. Further, we suggest the feasibility of our targeting method as a planning strategy for SRS of TRTN using a quantitative analysis of the radiation dose to adjacent vital structures.

\section{Methods}

\section{Patient Selection}

From January 1998 to December 2013, 280 patients with TN were treated with GKS at Seoul National University Hospital. Of these patients, those with TRTN were selected for analysis with approval from our institutional review board. The following inclusion criteria were used in this study: the patient 1) had an intracranial adjacent tumor on the trigeminal nerve that was confirmed by MRI; 2) had a typical TN on the side of the adjacent tumor, which was defined as an intense, sudden, shock-like, or stabbing episodic pain on the face along the unilateral division of the trigeminal nerve; ${ }^{5,7} 3$ ) was treated with GKS targeting both the tumor and trigeminal REZ in a single session; 4) had not undergone a previous transcranial resection of the tumor or microvascular decompression for neuralgic pain; 5) had visible trigeminal REZ on high-resolution MR images; and 6) had at least 1 year of clinical follow-up. Data from 15 consecutive patients who all underwent GKS in accordance with our selection criteria for GKS in TRTN were included in the present study.

Patients were considered for GKS for the following reasons: the patient 1) was 65 years of age or older, 2) had medical comorbidities such as severe cardiovascular disease or uncontrolled diabetes mellitus, and 3) wanted to defer surgical treatment. The median age of the patients was 67 years (range 45-79 years; 6 men and 9 women) at the time of GKS (Table 1). None of the included patients underwent a percutaneous procedure before GKS. The tumor type was diagnosed on the basis of radiological findings: 11 patients had meningioma, 3 vestibular
TABLE 1. Patient baseline characteristics and radiosurgical parameters

\begin{tabular}{lc}
\hline \multicolumn{1}{c}{ Characteristic } & Value \\
\hline Total no. of pts & 15 \\
\hline M/F ratio & $6: 9$ \\
\hline Median age in yrs (range) & $67(45-79)$ \\
\hline Median length of follow-up in mos (range) & $38(12-78)$ \\
\hline Clinical & $19(5-60)$ \\
\hline Radiological & 7 \\
\hline No. of pts w/ involved CN V division & 6 \\
\hline 1 division & 2 \\
\hline 2 divisions & $11(73)$ \\
\hline All divisions & $3(20)$ \\
\hline No. of pts w/ tumor type on MR images (\%) & $1(7)$ \\
\hline Meningioma & \\
\hline Vestibular schwannoma & 11 \\
\hline Trigeminal schwannoma & \\
\hline No. of pts w/ pre-GKS BNI score & $13(11-15)$ \\
\hline IV & $80(70-85)$ \\
\hline V & \\
\hline Median tumor vol in cm ${ }^{3}$ (range) & \\
\hline Median Rx dose in Gy (range) & \\
\hline To tumor & \\
\hline To trigeminal REZ & \\
\hline
\end{tabular}

pts $=$ patients; $R x=$ prescription .

schwannoma, and 1 trigeminal schwannoma. The median symptom duration before GKS was 6 months (range 1-50 months). All patient data were retrospectively collected using hospital chart review and telephone interviews with the patients.

\section{Stereotactic Radiosurgery}

All patients underwent a single-session SRS that targeted both the tumor and trigeminal REZ with the Leksell Gamma Knife Model Perfexion (Elekta Instrument AB). On the day of the treatment, the Leksell model $G$ frame was applied to the patient's head under conscious sedation and local scalp anesthesia. T1-weighted or T2-weighted, 3D, multiplanar, rapid-acquisition, gradient-echo MRI was used to obtain sequences with a slice thickness of $1.0 \mathrm{~mm}$ or $1.5 \mathrm{~mm}$ before gadolinium administration. Treatment planning was performed with the axial image supplemented with reconstructed coronal and sagittal images to enhance the accuracy with Gamma Plan software (Elekta Instrument AB). In all cases, the primary GKS targets were the tumor and the trigeminal REZ.

Multiple isocenters of the tumor were used to minimize radiation-related adverse effects on adjacent structures, including the brainstem and CNs VII-VIII. The median volume of the tumors was $1.7 \mathrm{~cm}^{3}$ (range $\left.0.1-4.9 \mathrm{~cm}^{3}\right)$. The following median prescription doses to the tumor, which were defined as a marginal dose at the $50 \%$ isodose line according to tumor type, were applied: $13 \mathrm{~Gy}$ (range 12.5-15 Gy) for meningioma, 12 Gy (range 11-13 Gy) for vestibular schwannoma, and 15 Gy for trigeminal schwannoma. 
The trigeminal REZ for targeting was defined as $2-4 \mathrm{~mm}$ anterior to the junction of the trigeminal nerve and the pons, and this area was irradiated using a single isocenter with a 4-mm collimator helmet. The median prescription dose, defined as maximal dose, to the trigeminal REZ was 80 Gy (range 70-85 Gy). Figure 1 shows an example of GKS planning and follow-up MRI examinations.

\section{Follow-Up Assessments}

Clinical and radiological follow-up examinations after GKS of most of our patients were performed according to our follow-up protocol for benign brain tumors and $\mathrm{TN}$ : clinical follow-up at $1,3,6$, and 12 months, with annual follow-ups thereafter, and radiological follow-up at 6, 12, 18 , and 24 months, with annual follow-ups thereafter. Two patients were lost during regular follow-up, and clinical follow-up data were obtained through telephone interviews with these patients. The median length of clinical follow-up after GKS was 38 months (range 12-78 months), and the median length of radiological follow-up was 19 months (range 5-60 months) in 13 patients for whom radiological data were available.

Neuralgic pain was assessed with the modified Barrow Neurological Institute (BNI) pain intensity score. ${ }^{18}$ The following scores for trigeminal pain were defined: Score I, no trigeminal pain without medication; Score II, occasional pain, not requiring medication; Score IIIa, no pain, continued medication; Score IIIb, persistent pain, but adequately controlled with medication; Score IV, some pain, not adequately controlled with medication; and Score V, severe pain/no pain relief. The initial BNI score was above Score IIIb in all patients. The patients were taking medications such as carbamazepine and gabapentin to alleviate the neuralgic pain. Pain improvement was defined as any improvement in the BNI pain intensity score compared with the initial BNI score. ${ }^{7}$ Failure of pain control was defined as a BNI score of IV or V without any improvement at the last follow-up after GKS. Pain recurrence was defined as any worsening in maximum pain after initial improvement during follow-up. Failure of tumor control was
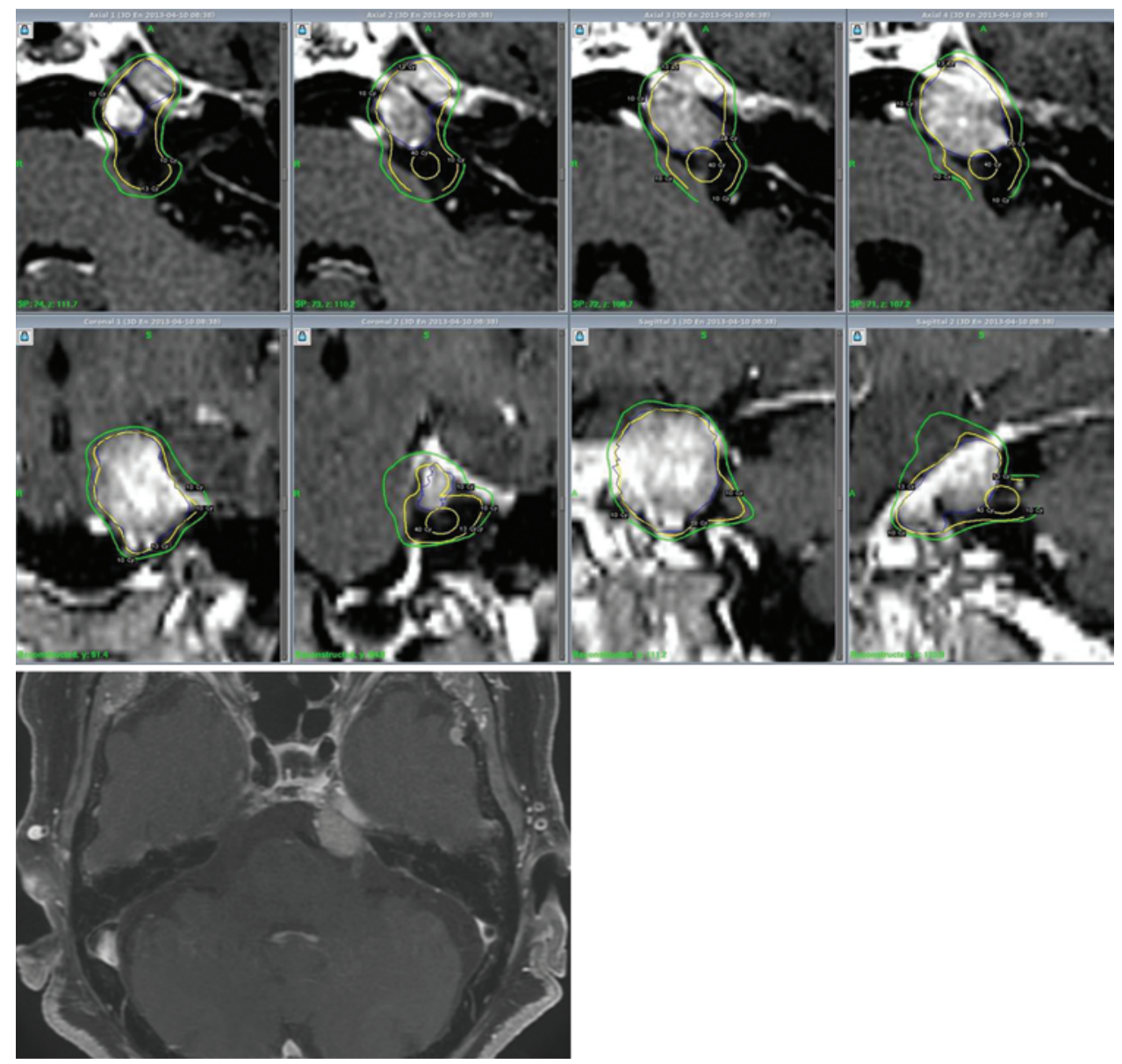

FIG. 1. GKS planning and follow-up MR images acquired in a 76-year-old woman with a well-demarcated mass in the left cerebellopontine angle. The radiological diagnosis of the tumor was meningioma, and the tumor had a volume of $4 \mathrm{~cm}^{3}$. Upper panels: The blue line delineates the tumor. The marginal dose of the tumor at the $50 \%$ isodose line (outer yellow line) was $13 \mathrm{~Gy}$. The inner yellow line is a 40-Gy line to cover the trigeminal REZ, and the maximal dose to the trigeminal REZ was 80 Gy. The green line is a 10-Gy line that was drawn to determine how much of the brainstem was included in the volume exposed to 10 Gy. The patient's neuralgic pain improved from BNI pain Score IV to I without any complications, including facial numbness. Lower panel: After GKS, the tumor size remained the same without increasing during a follow-up period of 18 months. Figure is available in color online only. 
defined as an increase in the initial tumor volume of more than $15 \%$ during the follow-up period..$^{20}$ Two patients who were lost to regular follow-up were excluded from radiological evaluations of tumor control because radiological data were unavailable for these patients.

The extent of facial numbness after GKS was evaluated with the BNI facial numbness score and considered a radiation dose-related adverse effect on the brainstem. ${ }^{17,25}$ None of the patients exhibited facial numbness before GKS. The following scores for numbness were defined: Score I, no facial numbness; Score II, mild facial numbness, but not bothersome; Score III, facial numbness, somewhat bothersome; and Score IV, facial numbness, very bothersome. All patients were also asked to describe their neurological status after GKS, especially about those faculties related to the function of the CN VII-VIII complex, including facial weakness, impaired taste function, hearing impairment, and balance difficulties. Three patients with vestibular schwannoma showed nonserviceable hearing before GKS, according to the American Academy of Otolaryngology-Head and Neck Surgery hearing classification system. ${ }^{11}$

\section{Dosimetry Analysis}

Quantitative analyses were performed to evaluate the effect of radiation dose on organs at risk (OARs), including the brainstem and the CN VII-VIII complex. Various parameters, including the radiation dose received by $2 \%$ or $50 \%$ of the tissue $\left(\mathrm{D}_{2 \%}\right.$ and $\mathrm{D}_{50 \%}$, respectively), and tissue volume covered by a dose of $12 \mathrm{~Gy}\left(\mathrm{~V}_{12 \mathrm{~Gy}}\right)$, were evaluated in the OAR analysis. Tumor coverage was defined as a dose that covered $95 \%$ of the tumor.

Dosimetry evaluations were conducted by a medical physicist who was blinded to the clinical information of each patient. All dosimetric data are presented as mean $\pm \mathrm{SD}$.

\section{Statistical Analysis}

The Kaplan-Meier method was used for analyses of actuarial recurrence-free survival rates for TN. The statistical analyses were performed with IBM SPSS Statistics software version 21.0 (SPSS IBM).

\section{Results \\ Clinical and Radiological Outcomes}

A comparison of the patients' BNI scores after GKS with those before GKS indicated initial neuralgic pain relief in $14(93.3 \%)$ of the 15 patients. The median length of time to maximal pain relief after GKS was 1 month (range 1-12 months), and in 9 patients (64.3\%), the neuralgic pain improved within 1 month. Failure of pain control was observed in 1 patient in whom MRI scans showed a petroclival meningioma (with a $3.2 \mathrm{~cm}^{3}$ volume); however, tumor control in this patient was observed at the last follow-up. The BNI scores at the time of the maximal response after GKS and the length of time to the maximal response are summarized in Table 2.

During the follow-up period, pain recurred in 3 (21.4\%) of the 14 patients who experienced pain relief after GKS: 1 patient's BNI score worsened from IIIa to IV, and the 2 other patients' scores increased from II to IIIb. How-
TABLE 2. Summary of clinical outcomes in the 15 patients after GKS

\begin{tabular}{|c|c|}
\hline Parameters & $\begin{array}{l}\text { No. of Patients } \\
(\%)\end{array}$ \\
\hline Initial pain relief & $14(93.3)$ \\
\hline \multicolumn{2}{|l|}{ BNI score* } \\
\hline 1 & 7 \\
\hline II & 5 \\
\hline Illa & 2 \\
\hline No response & 1 \\
\hline \multicolumn{2}{|l|}{ Time from GKS to max response } \\
\hline $1 \mathrm{mo}$ & 9 \\
\hline $3 \mathrm{mos}$ & 1 \\
\hline $6 \mathrm{mos}$ & 1 \\
\hline $1 \mathrm{yr}$ & 3 \\
\hline No response & 1 \\
\hline $\begin{array}{l}\text { Pain recurrence after initial pain relief during follow- } \\
\text { up period }\end{array}$ & $3(21.4)$ \\
\hline \multicolumn{2}{|l|}{ BNI score at last follow-up } \\
\hline 1 & 7 \\
\hline II & 3 \\
\hline IIla & 1 \\
\hline Illb & 2 \\
\hline IV & 2 \\
\hline Persistent new-onset facial numbness & $3(20.0)$ \\
\hline
\end{tabular}

* At the time of max response.

ever, in none of the patients was a failure of tumor control observed on follow-up MRI scans. The patient with pain recurrence and a BNI score of IV underwent several percutaneous radiofrequency thermocoagulations; however, the other 2 patients did not undergo any additional procedures, except for added medication-based treatment. The actuarial recurrence-free survival rates were 93\%, 83\%, and $69 \%$ at 1,3 , and 5 years after GKS, respectively (Fig. 2). No pain recurred in patients with a BNI score of I at the time of the maximal treatment response.

At the last follow-up, 10 patients (66.7\%) had pain relief without medication, and in 2 patients (13.3\%), GKS failed to control the neuralgic pain. Tumor control was achieved in 13 of the patients with available radiological data.

\section{Complications}

New-onset facial numbness developed in 4 patients (26.7\%) at varying points during 3-30 months of followup. One of these patients had a BNI facial numbness score of IV, and the other 3 patients had a score of II. One of the 3 patients fully recovered without aggravation of neuralgic pain. Facial numbness persisted in 3 patients $(20.0 \%)$ at the last follow-up. None of the patients reported symptoms related to impaired function of the CN VII-VIII complex such as facial weakness, altered taste function, hearing impairment, and balance difficulties. No new-onset radiological changes were observed in the brainstem or REZ on follow-up MRI scans, including T2-weighted sequences and fluid-attenuated inversion recovery sequences. 


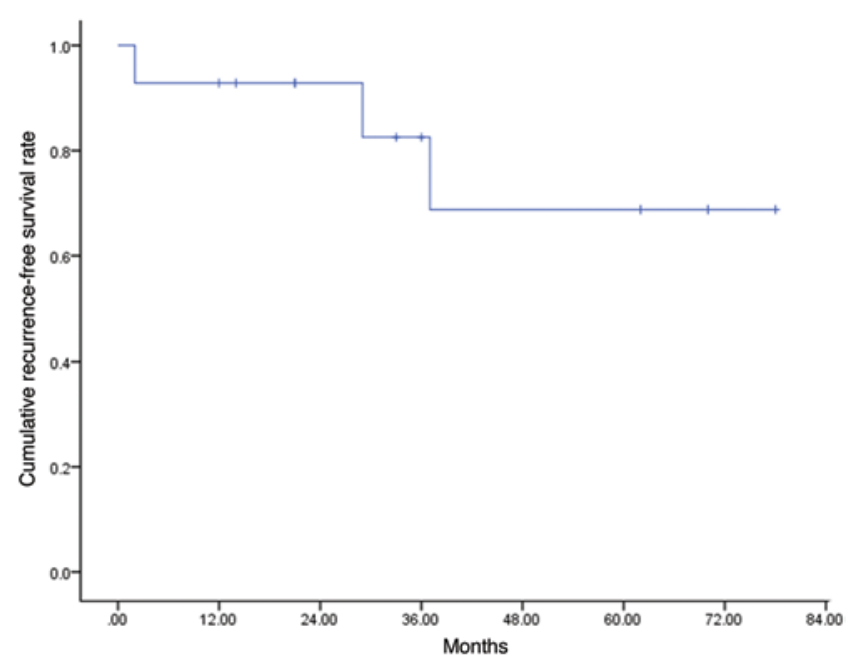

FIG. 2. Kaplan-Meier plot indicating cumulative recurrence-free survival for all patients; vertical tick marks on the plotted line indicate follow-up examinations. Figure is available in color online only.

\section{Results of Dosimetry Analyses}

Table 3 summarizes the dosimetric parameters used in our planning strategies for the selected OARs. The $\mathrm{V}_{12 \mathrm{~Gy}}$ to the brainstem was less than or equal to $0.24 \mathrm{~cm}^{3}$ in all patients. The $\mathrm{V}_{12 \mathrm{~Gy}}$ to the brainstem of patients who developed facial numbness after GKS was $0.15 \pm 0.08 \mathrm{~cm}^{3}$ (range $0.02-0.24 \mathrm{~cm}^{3}$ ), which was not statistically significantly different from that of patients without numbness $\left(0.15 \pm 0.01 \mathrm{~cm}^{3}\right.$, range $\left.0.13-0.16 \mathrm{~cm}^{3}\right)(\mathrm{p}=0.571)$. There were also no significant differences in radiation doses to other OARs between the patients with and those without facial numbness $\left(\mathrm{p}=0.489\right.$ for $\mathrm{D}_{2 \%}$ and $\mathrm{p}=0.571$ for $\left.\mathrm{D}_{50 \%}\right)$.

\section{Discussion}

Pharmacotherapy has been used as a first-line treatment modality in patients with idiopathic TN. ${ }^{5}$ Several invasive procedures such as radiofrequency thermorhizotomy and percutaneous balloon compression have also been considered for controlling pain in patients with medically intractable neuralgic pain or adverse effects related to medications. 5,22,24 These modalities can also be adapted for use in patients with TRTN; however, only temporary reductions in neuralgic pain are observed because of the pathophysiology of TRTN, in which the tumor directly compresses the trigeminal nerve. ${ }^{2,4}$ Therefore, resection of the adjacent tumor that is causing pain is likely the first-line choice for achieving durable pain control. This concept is reflected in the treatment of patients with TRTN with SRS; however, in previous reports, the primary target for SRS was limited to the tumor itself. 8,10,14,16,23

Researchers have previously considered various factors influencing the use of SRS for treating patients with TRTN. First, the vessel that is displaced by the tumor can be located near the trigeminal REZ, which acts as the offending vessel, similar to the etiology in idiopathic TN. ${ }^{2,9,22}$ Second, the relationship between reductions in tumor volume and pain control is controversial, and initial pain responses precede tumor shrinkage in most patients with TRTN after SRS. ${ }^{8}$ Kano et al. ${ }^{10}$ reported that tumor
TABLE 3. Summary of average dosimetric parameters for selected OARs*

\begin{tabular}{lcc}
\hline Parameter & Brainstem & CN VII-VIII Complex \\
\hline$D_{2 \%}$ in Gy & $10.05 \pm 1.38(6.35-11.50)$ & $9.71 \pm 5.36(4.15-19.40)$ \\
\hline$D_{50 \%}$ in $\mathrm{Gy}$ & $1.86 \pm 0.74(0.43-3.35)$ & $4.37 \pm 2.00(1.50-8.50)$ \\
\hline $\mathrm{V}_{12 \mathrm{~Gy}}$ in $\mathrm{ml}$ & $0.14 \pm 0.07(0.02-0.24)$ & $\mathrm{NA}$ \\
\hline $\mathrm{NA}=$ not applicable. \\
* Values represent mean $\pm \mathrm{SD}$ (range).
\end{tabular}

shrinkage was neither associated with pain relief nor with pain re-aggravation. Tanaka et al. ${ }^{23}$ also reported no detectable relationship between tumor shrinkage and pain relief. These findings imply that the strategy of targeting the tumor alone may be insufficient for durable pain control with SRS in patients with TRTN. To the best of our knowledge, the present study is the first report to evaluate the clinical outcomes of SRS when both the tumor and the trigeminal REZ are targeted in a single session.

Previous studies of SRS targeting of the tumor alone in TRTN patients have reported that the rate of initial pain response was $81 \%-83 \%$, and the rate of initial complete pain resolution without medication was 33\%-58\%., $8,10,14,21,23$ Facial pain in the present study was initially alleviated in 14 patients (93.3\%), which is a slightly better response compared with that described in previous reports. Our observed rate of initial complete pain resolution without medication (66.7\%) was also similar to that described in previous reports.

The durability of pain relief after treatment is important for improving the quality of life in TRTN patients. Recently, the largest series of SRS of TRTN was reported by Tanaka et al., ${ }^{23}$ who studied outcomes in 31 TRTN patients with benign brain tumors who were treated with SRS that targeted only the tumor and were followed up for at least 1 year. The authors observed pain recurrence in $7(46.7 \%)$ of 15 patients in whom the pain had initially completely resolved; the actuarial pain relief maintenance rate in that study was $80 \%$ at 1 year and $72 \%$ at 2 years after initial pain resolution. Squire and colleagues ${ }^{21}$ reported that the recurrence-free survival rate of TRTN patients with BNI scores of IV and V who were treated with SRS targeting only the tumor was $66 \%$ at 1 year and $53 \%$ at 2 years. In the present study, pain did not recur in patients with a BNI score of I at the time of the maximal response, and the actuarial recurrence-free survival rates were $93 \%$ at 1 year and $83 \%$ at 3 years. The rate of pain recurrence at the last follow-up was $21.4 \%$, which was also better than those previously reported $(24 \%-47 \%))^{8,10,14,21,23}$ It is difficult to objectively assess the discrepancy in clinical outcomes between our study and previous studies of TRTN management with SRS targeting the tumor only. However, it is plausible that SRS targeting both the tumor and trigeminal REZ in a single session achieves durable pain relief in patients with TRTN.

There is no tight relationship between radiation dose to the brainstem and facial numbness developing after SRS. ${ }^{1,6}$ Balagamwala et al. ${ }^{1}$ reported that facial numbness was not statistically significantly correlated with radiation dose and extent to OARs in the brainstem, especially 
not with the maximal dose and $\mathrm{V}_{12 \mathrm{~Gy}}$. In the present study, the rate of new-onset facial numbness was $20.0 \%$ at the last follow-up, which was slightly higher than the rates reported in previous studies of SRS targeting only mass lesions. ${ }^{10,14,21,23}$ Patients with new-onset facial numbness and patients without numbness after GKS did not significantly differ in $\mathrm{V}_{12 \mathrm{~Gy}}$ to the brainstem. However, a reduction in the radiation dose for trigeminal REZs may be considered in TRTN patients when performing SRS targeting both the tumor and the trigeminal REZ in a single session. The value of $\mathrm{V}_{12 \mathrm{~Gy}}$ in all patients was less than or equal to $0.24 \mathrm{~cm}^{3}$, which may be considered the cut-off value to avoid new-onset facial numbness in TRTN patients after single-session SRS targeting both the tumor and the trigeminal REZ.

The present study evaluated the clinical outcomes among TRTN patients with typical neuralgic pain who underwent no other treatment before GKS. Our method of targeting GKS to both the tumor and the trigeminal REZ in a single session can be also applied as an alternative treatment strategy to patients with tumor regrowth and recurrent pain after resection.

As with any retrospective study, this study has inherent biases and limitations. First, our sample size was relatively small, which may have caused a selection bias. Although TRTN is a rare disease entity, larger clinical trials or casematched studies are required to validate our results. Second, our dose plan can be applied only when the REZ is visible on high-resolution MRI scans. If the tumor causing the neuralgic pain is relatively small, its small size may interfere with the interpretation of several important outcomes, including pain control, and of the relationship between radiation dose and the development of facial numbness. Third, the clinical data of 2 patients lost to follow-up were obtained through a telephone interview, which may cause recall bias. Further investigations employing a prospective study design should be performed to validate the present results.

\section{Conclusions}

Single-session GKS for targeting both tumor and trigeminal REZ in treatments for TRTN represents an alternative and safe strategy for increasing initial pain reduction and improving the durability of pain relief without increasing radiation dose-related adverse effects in TRTN patients with visible trigeminal REZ. Radiation doses to the brainstem and to the circumjacent cranial nerves must be considered before GKS to avoid significant complications.

\section{References}

1. Balagamwala EH, Suh JH, Barnett GH, Khan MK, Neyman G, Cai RS, et al: The importance of the conformality, heterogeneity, and gradient indices in evaluating Gamma Knife radiosurgery treatment plans for intracranial meningiomas. Int J Radiat Oncol Biol Phys 83:1406-1413, 2012

2. Barker FG II, Jannetta PJ, Babu RP, Pomonis S, Bissonette DJ, Jho HD: Long-term outcome after operation for trigeminal neuralgia in patients with posterior fossa tumors. J Neurosurg 84:818-825, 1996

3. Bullitt E, Tew JM, Boyd J: Intracranial tumors in patients with facial pain. J Neurosurg 64:865-871, 1986
4. Cheng TM, Cascino TL, Onofrio BM: Comprehensive study of diagnosis and treatment of trigeminal neuralgia secondary to tumors. Neurology 43:2298-2302, 1993

5. Cruccu G, Gronseth G, Alksne J, Argoff C, Brainin M, Burchiel K, et al: AAN-EFNS guidelines on trigeminal neuralgia management. Eur J Neurol 15:1013-1028, 2008

6. Foote KD, Friedman WA, Buatti JM, Meeks SL, Bova FJ, Kubilis PS: Analysis of risk factors associated with radiosurgery for vestibular schwannoma. J Neurosurg 95:440-449, 2001

7. Han JH, Kim DG, Chung HT, Paek SH, Kim YH, Kim CY, et al: Long-term outcome of gamma knife radiosurgery for treatment of typical trigeminal neuralgia. Int J Radiat Oncol Biol Phys 75:822-827, 2009

8. Huang CF, Tu HT, Liu WS, Lin LY: Gamma Knife surgery for trigeminal pain caused by benign brain tumors. J Neurosurg 109 Suppl:154-159, 2008

9. Jannetta PJ: Treatment of trigeminal neuralgia by suboccipital and transtentorial cranial operations. Clin Neurosurg 24:538-549, 1977

10. Kano H, Awan NR, Flannery TJ, Iyer A, Flickinger JC, Lunsford LD, et al: Stereotactic radiosurgery for patients with trigeminal neuralgia associated with petroclival meningiomas. Stereotact Funct Neurosurg 89:17-24, 2011

11. Monsell EM: New and revised reporting guidelines from the Committee on Hearing and Equilibrium. Otolaryngol Head Neck Surg 113:176-178, 1995

12. Murovic JA, Chang SD: A critical analysis of the literature review in "Stereotactic radiosurgery for trigeminal pain secondary to benign skull base tumors" by Tanaka et al. and presentation of an algorithm for management of these tumors. World Neurosurg 80:287-289, 2013

13. Nomura T, Ikezaki K, Matsushima T, Fukui M: Trigeminal neuralgia: differentiation between intracranial mass lesions and ordinary vascular compression as causative lesions. Neurosurg Rev 17:51-57, 1994

14. Pollock BE, Iuliano BA, Foote RL, Gorman DA: Stereotactic radiosurgery for tumor-related trigeminal pain. Neurosurgery 46:576-583, 2000

15. Puca A, Meglio M, Vari R, Tamburrini G, Tancredi A: Evaluation of fifth nerve dysfunction in 136 patients with middle and posterior cranial fossae tumors. Eur Neurol 35:33-37, 1995

16. Régis J, Metellus P, Dufour H, Roche PH, Muracciole X, Pellet W, et al: Long-term outcome after gamma knife surgery for secondary trigeminal neuralgia. J Neurosurg 95:199205, 2001

17. Rogers CL, Shetter AG, Fiedler JA, Smith KA, Han PP, Speiser BL: Gamma knife radiosurgery for trigeminal neuralgia: the initial experience of The Barrow Neurological Institute. Int J Radiat Oncol Biol Phys 47:1013-1019, 2000

18. Rogers CL, Shetter AG, Ponce FA, Fiedler JA, Smith KA, Speiser BL: Gamma knife radiosurgery for trigeminal neuralgia associated with multiple sclerosis. J Neurosurg 97 (5 Suppl):529-532, 2002

19. Sivakanthan S, Van Gompel JJ, Alikhani P, van Loveren $\mathrm{H}$, Chen R, Agazzi S: Surgical management of trigeminal neuralgia: use and cost-effectiveness from an analysis of the Medicare Claims Database. Neurosurgery 75:220-226, 2014

20. Snell JW, Sheehan J, Stroila M, Steiner L: Assessment of imaging studies used with radiosurgery: a volumetric algorithm and an estimation of its error. Technical note. J Neurosurg 104:157-162, 2006

21. Squire SE, Chan MD, Furr RM, Lowell DA, Tatter SB, Ellis TL, et al: Gamma knife radiosurgery in the treatment of tumor-related facial pain. Stereotact Funct Neurosurg 90:145-150, 2012

22. Tan LA, Gerard CS, Ahuja SK, Moftakhar R: Retrosigmoid approach for resection of cerebellopontine angle meningioma 
and decompression of the trigeminal nerve. Neurosurg Focus 36 (1 Suppl):1, 2014

23. Tanaka S, Pollock BE, Stafford SL, Link MJ: Stereotactic radiosurgery for trigeminal pain secondary to benign skull base tumors. World Neurosurg 80:371-377, 2013

24. Tatli M, Satici O, Kanpolat Y, Sindou M: Various surgical modalities for trigeminal neuralgia: literature study of respective long-term outcomes. Acta Neurochir (Wien) 150:243-255, 2008

25. Xu Z, Schlesinger D, Moldovan K, Przybylowski C, Sun X, Lee CC, et al: Impact of target location on the response of trigeminal neuralgia to stereotactic radiosurgery. J Neurosurg 120:716-724, 2014

\section{Disclosures}

The authors report no conflict of interest concerning the materials or methods used in this study or the findings specified in this paper.

\section{Author Contributions}

Conception and design: DG Kim, JW Kim. Acquisition of data: SK Kim, Se. Analysis and interpretation of data: DG Kim, SK Kim, Chung. Drafting the article: SK Kim. Critically revising the article: SK Kim, Chung. Reviewed submitted version of manuscript: DG Kim, Se, JW Kim, YH Kim, Chung, Paek. Approved the final version of the manuscript on behalf of all authors: DG Kim. Statistical analysis: SK Kim. Administrative/technical/material support: DG Kim, YH Kim, Paek. Study supervision: DG Kim.

\section{Supplemental Information}

Previous Presentations

Portion of this work were presented in abstract form at the 17th Leksell Gamma Knife Society Annual Meeting, New York City, New York, May 11-15, 2014.

\section{Current Affiliations}

Dr. S. K. Kim: Department of Neurosurgery, Gyeongsang National University School of Medicine and Gyeongsang National University Hospital, Jinju, Republic of Korea.

\section{Correspondence}

Dong Gyu Kim, Department of Neurosurgery, Seoul National University Hospital, Seoul National University College of Medicine, 101 Daehak-ro Jongno-gu, Seoul 110-744, Korea. email: gknife@snu.ac.kr. 